\title{
Surgical Robot Control Based on Torque Control Method
}

\author{
Bin Zhao ${ }^{1, a}$, Xusheng Shi ${ }^{2, b}$ \\ ${ }^{1}$ School of Automation Science and Electrical Engineering, Beihang University, 100191, Beijing, \\ PRC \\ ${ }^{2}$ Advanced Manufacturing Technology and Systems Research Center, Department of Industrial \\ and Manufacturing Systems Engineering, Beihang University, 100191, Beijing, PRC \\ a173402910@qq.com, bshixu1214@163.com
}

Keywords: Surgical Robot; Torque control; Adaptive control

\begin{abstract}
Surgical robots are usually controlled by the method of the independent PD or the PD model based on gravity compensation. However, the system is still a open loop control with both of the control models. The two models can not consider the dynamics effectively either. The position error can not be guaranteed. To solve the problem, a torque control model based on dynamic model and adaptive model is proposed. And the position tolerance of PD control based on gravity compensation and that of control based on Torque Control stability theory are compared by Matlab software. The maximal tolerance of the new method is red robot end-effector is $0.15 \mathrm{~mm}$.
\end{abstract}

\section{Introduction}

The robotic system is a redundancy, nonlinear dynamic system[1]. The control of the robot is basically talking about the motion and status of the end-effectors. The position control and trajectory tracking are two main categories of the control[2].

The control methods of robotic could be categorized into the kinematic and dynamic methods. The kinematic methods are usually deployed for the motion control. The independent PD control algorithm skips the dynamic model of the robot. The position feedback were compared to the desire value in order to eliminate the error[3]. A more practical method considered the weight of the robot. The gravity compensation could improve the control accuracy.

Both of the algorithms are linear equation based methods which are unable to reflect the nonlinear robotic system[4].

A torque control algorithm based on Dynamic model is proposed. The algorithm calculates the torque of the driving motors instead of position and trajectory. The controller is closed loop with a nonlinear differential equation of the position error.

\section{The Robotic and the Modeling using Sim-Mechanics}

The surgical robot in this research is a 6 DOF limb, as fig. 1. the first one is the prismatic joint. The second to the sixth joint sare rotational joints.

The Matlab SimMechanics was used for the robotic modeling. The simulation model shows as fig. $2^{[5]}$.

\section{Dynamic modeling}

Newton-Euler method and Lagrange equation are two major method for dynamic calculation of the surgical robot ${ }^{[6]}$. The Lagrange equation was used in this research, as Eq.1.

$\mathrm{L}$ is lagrange function. $\mathrm{K}$ is the kinetic energy and $\mathrm{P}$ is potential energy of the robot. 


$$
\begin{aligned}
& L=K-P=\frac{1}{2} \sum_{i=1}^{n} \sum_{j=1}^{i} \sum_{k=1}^{i} \operatorname{Trace}\left(\frac{\partial T_{i}}{\partial q_{i}} I_{i} \frac{\partial T_{i}^{T}}{\partial q_{k}}\right) \\
& \times \dot{q}_{j} \dot{q}_{k}+\frac{1}{2} \sum_{i=1}^{n} I_{a i} \dot{q}_{i}^{2}+\sum_{i=1}^{n} m_{i} g^{T} T_{i}{ }^{i} r_{i}, n=1,2, \ldots
\end{aligned}
$$

The $T_{i}$ is the relative coordination transformational matrix of ith joint. $I_{a i}$ is the equivalent moment of inertia. $\dot{q}_{j}$ is the velocity of the jth joint. the dynamic model of the robot shown as Eq. 2 and Eq. $3^{[2]}$.

$$
\begin{gathered}
T_{i}=\sum_{j=i}^{n} \sum_{k=1}^{j} \operatorname{Trace}\left(\frac{\partial T_{j}}{\partial q_{k}} I_{j} \frac{\partial T_{j}^{T}}{\partial q_{i}}\right) \ddot{q}_{k}+I_{a i} \ddot{q}_{i}+\sum_{j=1}^{n} \sum_{k=1}^{j} \sum_{m=1}^{j} \operatorname{Trace}\left(\frac{\partial^{2} T_{i}}{\partial q_{k} \partial q_{m}} I_{j} \frac{\partial T_{j}^{T}}{\partial q_{i}}\right) \dot{q}_{k} \dot{q}_{m}-\sum_{j=1}^{n} m_{j} g^{T} \frac{\partial T_{i}}{\partial q_{i}}{ }^{i} r_{i} \quad \text { (Eq.2) } \\
H(q) \ddot{q}+C(q, \dot{q}) \dot{q}+G(q)=\tau \quad \text { (Eq.3) }
\end{gathered}
$$

$q, \dot{q}, \ddot{q}$ are the position, velocity and acceleration of each joint respectively. $H(q) \ddot{q}$ is the inertia force based on generalized acceleration $\ddot{q} . C(q, \dot{q}) \dot{q}$ is quadric form of generalized velocity. $\dot{q}_{i}^{2}$ is the centrifugal force. $\dot{q}_{i} \dot{q}_{j}(i \neq j)$ is coriolis force. And $G(q)$ is the gravity force. $\tau$ is the driving force of each actuator ${ }^{[7]}$.

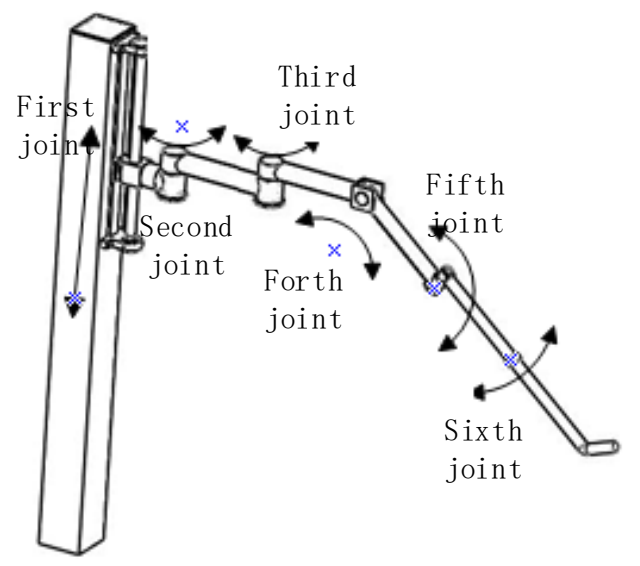

Fig.1 The surgical robot in this research is a 6 DOF limb

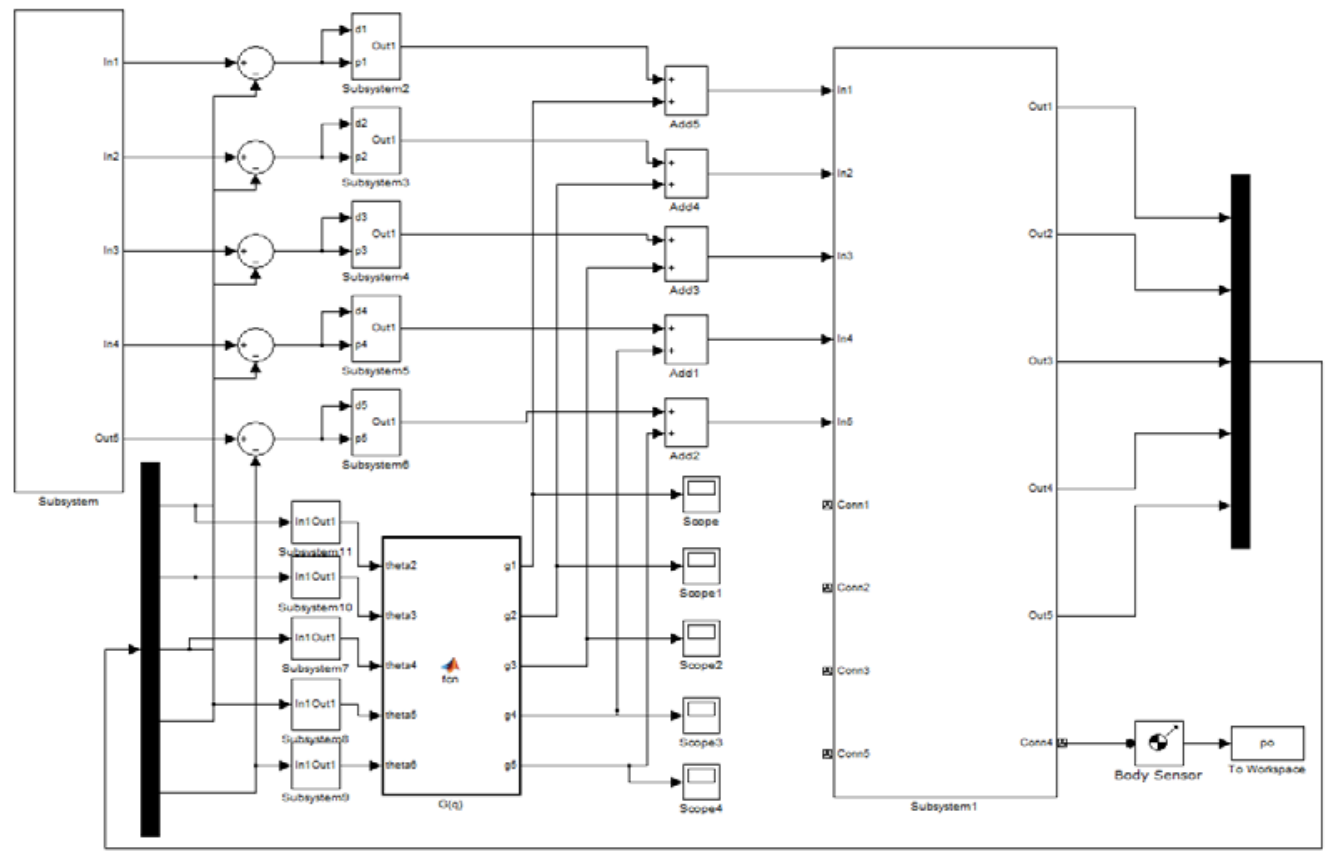

Fig.2 The model of Torque Controller 


\section{Control modeling}

Robotic is the nonlinear time-variant system. So, the functional analysis was used. The goal of the robot is trajectory tracking, which is a time-variant $q_{d}(t)$. And the position error: $e=q_{d}-q$.

$$
\dot{q}_{r}=\dot{q}_{d}+\Lambda\left(q_{d}-q\right)=\dot{q}_{d}+\Lambda e
$$

$\Lambda$ is the positive definite matrix. The control law is:

$$
\tau=H(q) \ddot{q}_{r}+C(q, \dot{q}) \dot{q}_{r}+G(q)+K_{d}\left(\dot{q}_{r}-\dot{q}\right)
$$

$K_{d}$ is the positive definite matrix. And the close loop equation will be:

$$
H(q) \ddot{q}+C(q, \dot{q}) \dot{q}+G(q)=H(q) \ddot{q}_{r}+C(q, \dot{q}) \dot{q}_{r}+G(q)+K_{d}\left(\dot{q}_{r}-\dot{q}\right)
$$

and

considered:

$$
H(q)\left(\ddot{q}_{r}-\ddot{q}\right)+C(q, \dot{q})\left(\dot{q}_{r}-\dot{q}\right)+K_{d}\left(\dot{q}_{r}-\dot{q}\right)=0
$$

So:

$$
\dot{q}_{r}-\dot{q}=\dot{e}+\Lambda e, \ddot{q}_{r}-\ddot{q}=\ddot{e}+\Lambda \dot{e}
$$

$$
H\left(q_{d}-e\right) \ddot{e}+\Lambda \dot{e}+C\left(q_{d}-e, \dot{q}_{d}-\dot{e}\right)(\dot{e}+\Lambda e)+K_{d}(\dot{e}+\Lambda e)=0
$$

The torque control algorithm uses the dynamic model of the robot. And it is a close loop with a nonlinear differential equation for the error $e^{[4]}$.

\section{Simulation and Verification}

The trajectory of the surgical robot from $\mathrm{A}(0,1402.7,878.7)$ to $\mathrm{B}(-193.5,1410.8,914.1)$ was derived by Sim-Mechanics and inverse kinematic method. So the desire trajectory could be gotten,

The PD controller with gravity compensation considered the weight of all limbs and actuators. The close loop control was formed by adding the gravity feedback. The desire trajectory and result were compared in the fig.3.

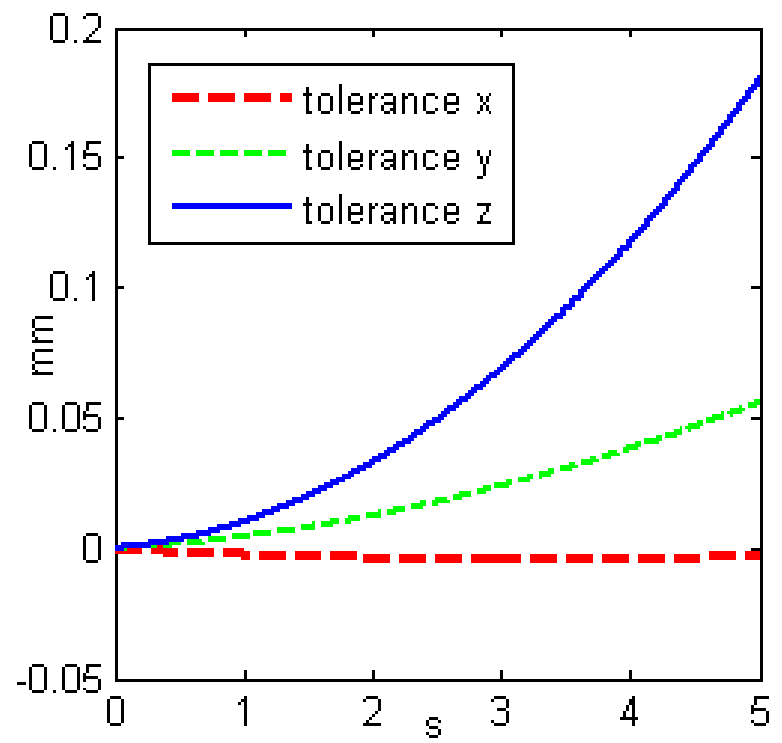

Fig.3 position error of robot based on PD controller with gravity compensation

The PD controller with gravity compensation is fast response and high accuracy. This algorithm achieved its best by using linear modeling to solve the nonlinear system. This research proposed the torque control algorithm. So the dynamic model will be:

$$
\tau=H(q) \ddot{q}_{r}+C(q, \dot{q}) \dot{q}_{r}+G(q)+K_{d}\left(\dot{q}_{r}-\dot{q}\right)
$$

and $\dot{q}_{r}=\dot{q}_{d}+\Lambda\left(q_{d}-q\right), \Lambda=\operatorname{diag}[15,0.2,40.1,4,2], K_{d}=\operatorname{diag}[0.1,0.2,0.045,0.06,0.1]$.

A more straight forward results comparison of the torque controller and PD controller shows as Fig.4. Fig. 4 shows the maximum error with torque controller is about $0.15 \mathrm{~mm}$, which is a better result than other methods. 


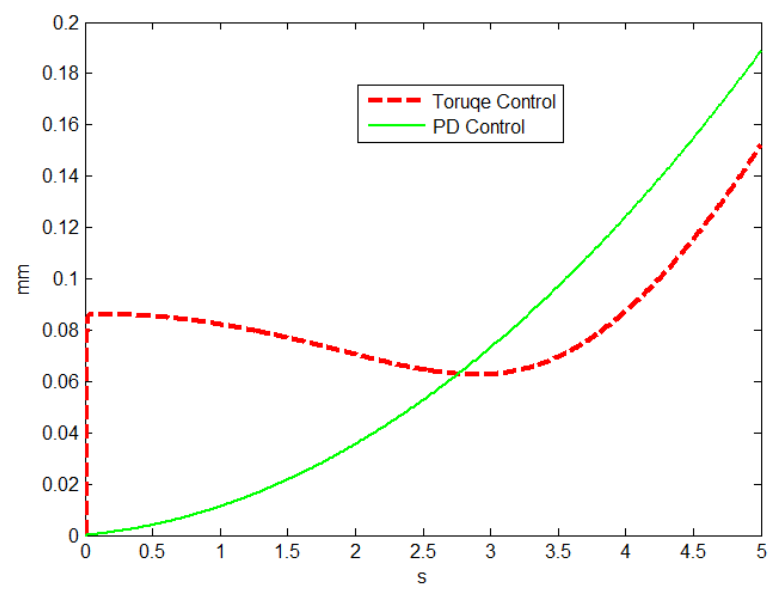

Fig.4 Tolrance Comparison of two control method The position error shown in Fig.5.

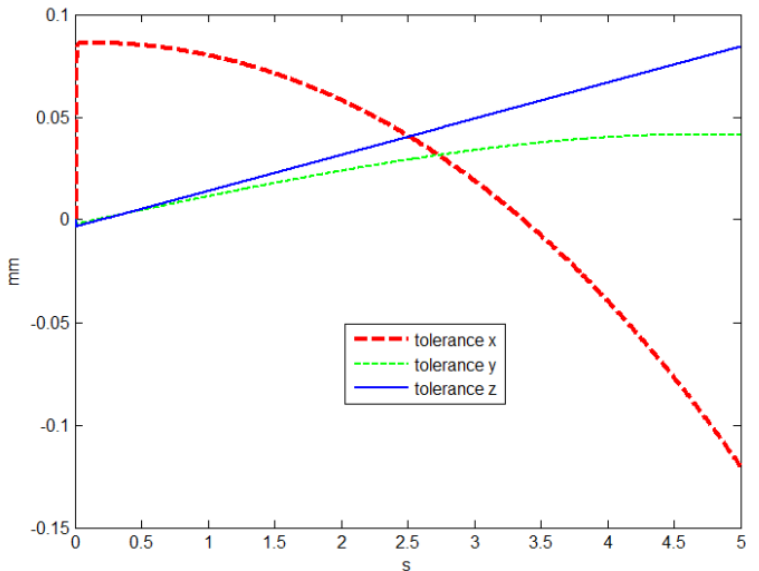

Fig.5 Position error by torque controller

\section{Conclusion}

A control algorithm based on Lagrange Equation and troque control were proposed. The dynamic model were considered in the new method. And the controller is closed loop with a nonlinear differential equation of the position error. The simulaition result proved the new algorithm provided a more accuracy in position control and trajectory tracking.

The Matrix $\Lambda$ and $K_{d}$ in the control model could be finely tuned to improve the PD controller. And a better control quality could be achieved.

\section{Reference}

[1] Gao Yunfeng, Lv Mingrui, Zhou Lun et al. Analysis of kinematics of a five-axis hybrid manipulator[J] Journal of Harbin Institure of technology. 2014,46(7):2-7.

[2] Chen Gang, Jia Qingxuan, Li Tong et al. Recursive Calibrations for Robot Kinematics Parameters[J], Journal of Beijing University of Posts and Telecommunications,2013,36(2): 28-32.

[3] $\mathrm{Hu}$ Shengbin, Lu Minxun. Fuzzy Integral Sliding Mode Control for Three-Links Spatial Robot[J]. Computer Simulation,2012,29(2):162-166.

[4] Yu Lingtao, Zhang Nan, Zhang Li-xun, et al. A Sim-Mechanics simulation of static forces in a 3-RPS parallel robot[J] Journal of Harbin Engineering University , 2010, 31(8): 1061-1064.

[5] Gao Wenbin, Wang Hongguang, Jiang Yong, et al. Kinematic Calibration Method of Robots Based on Distance Error[J]. ROBOT,2013,35(5):600-606.

[6] Zhang Li-xun, SUN Hong-ying, QIAN Zhen-mei. Kinematics Analysis and Simulation of Horizontal Lower Limbs Rehabilitative Robot[J]. Journal of System simulation. 2010, 22(8): 2001-2005. 